\title{
Terapia Morfoanalítica nos Transtornos de Sintomas Somáticos
}

\author{
Cristina Elena Prado Teles Fregonesi ${ }^{1}$ \\ ${ }^{1}$ Universidade Estadual Paulista Júlio de Mesquita \\ Filho, SP, Brasil.
}

\author{
Lazslo Antonio Ávila ${ }^{2}$ \\ ${ }^{2}$ Faculdade de Medicina de São José do Rio Preto, \\ SP, Brasil.
}

Resumo: A Terapia Morfoanalítica (TM) é uma terapia psicocorporal analítica que possibilita a integração das experiências psicoafetivas a partir do corpo. Permite compreender o componente psíquico da manifestação física, bem como a somatização do fenômeno psíquico. Este estudo objetivou avaliar o efeito da TM em pessoas com transtornos de sintomas somáticos, além de observar o impacto desta terapia na vida pessoal destes indivíduos. Trata-se de um Estudo de Caso de dois indivíduos. Foram realizadas 24 sessões de TM, uma vez por semana, com duração de uma hora. As avaliações constituíram-se de coleta de dados pessoais e antropométricos, nível de dor e flexibilidade muscular, bem como avaliação da presença de ansiedade, depressão e alexitimia, por meio de questionários. Também foi realizada avaliação da modulação autonômica. A evolução psicoemocional foi acompanhada no decorrer de todo tratamento morfoanalítico. A eficácia da TM foi observada tanto nos índices emocionais, quanto físicos. Isso demonstra a efetividade desta modalidade terapêutica, que associa os trabalhos corporais com o trabalho verbal analítico, na melhor integração psicossomática.

Palavras-chave: Terapias mente-corpo, Sintomas inexplicáveis, Frequência cardíaca.

\section{Morphoanalytic Therapy in Somatic Symptom Disorders}

\begin{abstract}
Morphoanalytic Therapy (MT) is an analytical psycho-corporal therapy that enables the integration of psycho-affective experiences from the body. It allows us to understand the psychic component of physical manifestation as well as the somatization of the psychic phenomenon. This study aimed to evaluate the effect of MT in people with somatic symptom disorder, in addition to observe the impact of this therapy on the personal life of these individuals. This is a case study of two individuals. Twenty-four MT sessions were performed once a week, lasting one hour each. The evaluations consisted of personal and anthropometric data collection, pain level and muscular flexibility, as well as of the presence of anxiety, depression and alexithymia, through questionnaires. Autonomic modulation was also evaluated. The psychoemotional evolution was followed during all morphoanalytic treatment. The efficacy of MT was observed in emotional and physical indexes. This demonstrates the effectiveness of this therapeutic modality, which associates bodily works with analytical verbal work, in the best psychosomatic integration.
\end{abstract}

Keywords: Mind-Body Therapies, Medically Unexplained Symptoms, Heart rate. 


\title{
Terapia Morfoanalítica en los Trastornos de Síntomas Somáticos
}

\begin{abstract}
Resumen: La Terapia Morfoanalítica (TM) es una terapia psico-corporal analítica que posibilita la integración de las experiencias psico-afectivas desde el cuerpo. Permite la comprensión del componente psíquico de la manifestación física, así como la somatización del fenómeno psíquico. Este estudio objetivó evaluar el efecto de la TM en personas con trastorno de síntomas somáticos, además de observar el impacto de esta terapia en la vida personal de estos individuos. Se trata de un estudio de caso de dos individuos. Se realizaron 24 sesiones de TM, una vez por semana, con una duración de una hora. Las evaluaciones se constituyeron de recolección de datos personales y antropométricos, nivel de dolor y flexibilidad muscular, así como evaluación de la presencia de ansiedad, depresión y alexitimia, por medio de cuestionarios. También se realizó una evaluación de la modulación autonómica. La evolución psicoemocional fue acompañada en el transcurso de todo tratamiento morfoanalítico. La eficacia de la TM fue observada tanto en los índices emocionales, como físicos. Esto demuestra la efectividad de esta modalidad terapéutica, que asocia los trabajos corporales con el trabajo verbal analítico, en la mejor integración psicosomática.
\end{abstract}

Palabras clave: Terapias mente-cuerpo, Síntomas inexplicables, Frecuencia cardiaca.

\section{Introdução}

O corpo humano, objeto passível de manifestações em diversificadas áreas do conhecimento e/ou meios culturais, é multifacetado e marcado pela complexidade. Nele está impressa toda vivência do sujeito. Todo o conjunto de fatores de ordem extracorporal também confluem para esse mesmo corpo. O corpo, fruto das particulares injunções no espaço-tempo, é objeto transdisciplinar por excelência. Este viabiliza a expressão de manifestações físicas e psíquicas, sendo, ao mesmo tempo, sujeito-objeto, fonte e finalidade da pulsão, biológico, cognitivo, erógeno, energético, imaginário, religioso e místico - corpo que nasce e corpo que morre (Ávila, 2012).

Porém, esse mesmo corpo constitui-se como fonte de sofrimento, frustração e dor. Em patologias da ação e do corporal, esse sofrimento parece encontrar dificuldades para se manifestar em termos psíquicos (Fernandes, 2011).

Neste contexto, quando uma questão subjetiva não ascende para a mente, como processo mental, ela pode se expressar no corpo e este adoece, gerando um conjunto de sintomas nos quais a etiologia orgânica está descartada "O processo somático ocupa o lugar do processo psíquico: no sintoma psicossomático uma questão subjetiva se apresenta, ao invés de representar" (Ávila, 2002).
Esses sintomas, associados ao sofrimento mental, não apresentam explicações médicas (Tófoli, Andrade, \& Fontes, 2011). Na década de 1980, sob a categoria diagnóstica "Transtornos Somatoformes", foram introduzidos nas classificações internacionais de doenças (Angenendt, \& Härter, 2015). Apresentam causa multifatorial e uma variedade de expressões desde estresse leve a estados de profunda debilidade - frequentemente associadas a quadros psiquiátricos de ansiedade e depressão (Angenendt, \& Härter, 2015). Caracterizam-se como um problema mundial, lotando serviços de saúde e gerando altos custos (Angenendt, \& Härter, 2015). Porém, decorrente da sobreposição e dificuldade diagnóstica desses transtornos, a classificação atual do DSM-V substituiu a terminologia transtornos somatoformes por transtorno de sintomas somáticos e transtornos relacionados. "A nova classificação define o diagnóstico principal, transtorno de sintomas somáticos, com base em sintomas positivos (sintomas somáticos perturbadores associados a pensamentos, sentimentos e comportamentos anormais em resposta a esses sintomas)" (American Psychiatric Association [APA], 2014).

Já o sistema de Classificação Internacional de Doenças (CID-10), no capítulo V sobre transtornos mentais e comportamentais, F45 - Transtornos Somatoformes, preconiza estes como sintomas físicos persistentes, sem base física. Quando presentes, não 
explicam a natureza e a extensão dos sintomas ou a angústia e preocupação do paciente (World Health Organization [WHO], 2016).

Assim, observa-se uma reconhecida participação dos fatores emocionais na manifestação somática (Heidari, Feizi, Roohafza, Hassanzadeh, \& Adibi, 2017). O corpo manifesta os sintomas, mas fatores psicológicos estão envolvidos ou em sua gênese ou no transcurso (Heidari et al., 2017).

Desta forma, para o tratamento de pacientes que adoeceram de corpo e alma, a elaboração de conexões entre diferentes manifestações da vida mental com suas expressões corporais psicossomáticas propicia um declínio desses sintomas "O significado de uma investigação psicossomática é procurar as pontes, tantas vezes perdidas, entre os corpos físico e vivido" (Ávila, 2012).

Partindo desta via de mão dupla entre "corpo físico e vivido", a inclusão da escuta do corpo sensível do paciente é fator relevante no atendimento de psicopatologias, como as psicossomatizações, possibilitando a manifestação da memória corporal no lugar da linguagem verbal. "A mobilização dessa memória corporal possibilita a representação psíquica de situações traumáticas registradas no corpo, e pode contribuir para reverter quadros psicopatológicos" (Fontes, 2006).

As interpretações dos significados das informações, assim como as reações secundárias a elas, são dependentes das ações conscientes e inconscientes comandadas ou mediadas pelo sistema nervoso central. Porém, a bagagem de informações de um indivíduo não está vinculada apenas em áreas específicas do cérebro, mas sim em todos os tecidos inervados e integrados. Tecidos corporais, como as fáscias musculares, possuem feixes colagenosos cristalinos com propriedades de condutância elétrica e piezoelétrica e esta característica tecidual produz um padrão de vibração informacional, que pode ser o responsável pelo elo existente entre a consciência corporal e a consciência cerebral (Souza, 2016).

Neste contexto, a Terapia Morfoanalítica (TM) - uma terapia psicocorporal analítica - possibilita, a partir do corpo, a integração das experiências psicoafetivas. Permite compreender, por um lado, o componente psíquico da manifestação física e, por outro, a somatização do fenômeno psíquico (Diefenbach, 2003; Pachioni, Fregonesi, \& Mantovani, 2014). Esta técnica utiliza conceitos das cadeias musculares de Françoise Mezieres (Pachioni et al., 2014) e da psi- canálise, baseado nos autores Jean Sarkissoff, Freud, Winnicott e Melaine Klein (Mignard, 2010; Pachioni, Palma, Mantovani, \& Fregonesi, 2016).

Como o bem-estar psicológico está associado ao desempenho cardiovascular (Krygier et al., 2013), visto que as alterações da variabilidade da frequência cardíaca (VFC) estão relacionadas ao estresse e à tensão emocional (Kuehl et al., 2015), a avaliação da modulação autonômica pode contribuir para o conhecimento do estado interno do sujeito. "O sistema nervoso autônomo não é tão autônomo assim e se encontra regulado pelas estruturas límbicas junto com o controle emocional" (Castro, Andrade, \& Muller, 2006).

A incapacidade em identificar, descrever e interpretar estados emocionais é referida como alexitimia, que significa "sem palavras para sentimentos" (Chalah, \& Ayache, 2017). Essa inabilidade em reconhecer as emoções de maneira adequada pode expor o indivíduo ao sofrimento psicológico (Margalit, Ben Har, Brill, \& Vatine, 2014) e consequente risco de desenvolver ansiedade e/ou depressão, bem como somatização (Hozoori \& Barahmand, 2013). Além disso, os processos emocionais são capazes de influenciar a respostas de dor quanto à percepção e intensidade (Tang, 2017), por isso a importância da regulação emocional nos tratamentos em geral. A TM trabalha essa globalidade.

Assim, o presente estudo tem como objetivo avaliar o efeito da Terapia Morfoanalítica em pessoas com transtornos de sintomas somáticos, por meio da análise da modulação autonômica, do nível de dor e flexibilidade muscular, da avaliação da presença de ansiedade e depressão e investigação da presença de alexitimia, além de observar o impacto desta terapia na vida pessoal destes indivíduos, acompanhando a evolução do indivíduo no decorrer de todo tratamento morfoanalítico.

\section{A Terapia Morfoanalítica}

A TM foi idealizada pelo fisioterapeuta francês Serge Peyrot na década de 1980 (Diefenbach, 2003; Peyrot, Mignard, \& Albuquerque, 2005). Este, após formação direta com Françoise Mézières, fisioterapeuta francesa que revelou o princípio terapêutico da globalidade do corpo, a partir dos conceitos das cadeias musculares (Pachioni et al., 2016), observou que as características exclusivamente biomecânicas não eram suficientes para compreender e analisar 
os sinais e sintomas consequentes aos desequilíbrios posturais. A partir de vivências clínicas posturais, observou a expressão de emoções e sentimentos em seus pacientes e propôs a complementação das intervenções biomecânicas com o trabalho analítico. Assim, criou a TM, integrando o trabalho corporal, com o trabalho verbal analítico e relacional, em todas as fases da sessão (Diefenbach, 2003; Peyrot et al., 2005).

Partindo do princípio da unidade psicocorporal, a TM trabalha reorganizando simultaneamente as estruturas musculares, psicoafetivas, neuromotoras e os esquemas sensoriais (Livorato, 2004). Conta com técnicas que atuam, concomitantemente, em três pilares que integram essa unidade: o trabalho no corpo real, no corpo vivenciado/sensorial e no corpo emocional/afetivo (Diefenbach, 2003; Livorato, 2004; Peyrot et al., 2005). O toque e a empatia, elementos fundamentais da construção do EU e parte da comunicação infraverbal, permeiam todo o quadro terapêutico, abrindo possibilidade para a expressão de sensações e sentimentos.

O corpo real é o corpo da pele, músculos, ligamentos, articulações, ossos e órgãos essenciais. O trabalho é realizado principalmente nos sistemas osteomuscular e tegumentar. As técnicas corporais permitem a reorganização da estrutura, com subsequente conscientização do conteúdo inconsciente das tensões. Podem ser utilizadas posturas Mezieristas de alongamento, associadas a trabalho respiratório, com enfoque particular na liberação do diafragma, centro das tensões musculares e emocionais. Também podem ser utilizadas massagens superficiais, com toques suaves e envolventes, ativando receptores cutâneos, e massagens profundas, atuando diretamente sobre o tecido conjuntivo e tensões musculares (Diefenbach, 2003; Livorato, 2004; Peyrot et al., 2005).

O corpo sensorial ou vivenciado é o corpo subjetivo em que o sujeito tem a concepção de ser e ter um lugar para as sensações e percepções. Embora este não corresponda, necessariamente, ao corpo real, é a partir desse que cada um se representa internamente e se relaciona no mundo. Para o corpo sensorial são utilizadas as técnicas de consciência corporal no qual o sistema proprioceptivo e exteroceptivo são despertados (Diefenbach, 2003; Livorato, 2004; Peyrot et al., 2005).

O corpo emocional ou afetivo contém o registro da história psicoafetiva. Armazena experiências e traumatismos vivenciados. Essa memória emocional está inscrita principalmente nos músculos e pele, que permitem inter-relações com o mundo externo. (Diefenbach, 2003; Livorato, 2004; Peyrot et al., 2005).

A sessão inicia-se com a leitura espontânea, na qual o paciente fica em pé de frente ao terapeuta, sendo questionado por este sobre suas percepções físicas e emocionais. Posteriormente, o paciente é convidado a ficar em decúbito dorsal e a proposta do trabalho corporal é escolhida em função da queixa física e/ou psíquica no momento da sessão. As sessões apresentam ritmo e dinâmica em função do momento que o paciente atravessa dentro do seu processo terapêutico. Pode-se propor um trabalho postural global, trabalho respiratório, massagens superficiais ou profundas, massagens para mobilização de líquidos corporais e manobras de lifting cervical, de membros superiores ou inferiores. Ao final, na posição em pé, é realizada uma nova consciência corporal onde o paciente percebe novas referências corporais e psíquicas e ocorre uma reavaliação da postura corporal global e análise dos aspectos psíquicos relatados pelo paciente.

O terapeuta morfoanalista ajuda o indivíduo a conscientizar-se do significado inconsciente do sintoma, parte da linguagem do corpo, dando lugar à expressão emocional. Este orienta o trabalho segundo as necessidades físicas e psíquicas do paciente, as intensidades das dores, o estado mais ou menos regressivo e a comunicação verbal e infraverbal do momento (Peyrot et al., 2005). A musculatura é vista como veículo de expressão ou repressão de sentimentos e os trabalhos propostos podem revelar esses conteúdos psíquicos. A qualidade de presença do terapeuta, o olhar, as palavras e o silêncio são elementos relevantes no tratamento (Diefenbach, 2003). Cada vez que o paciente coloca sua presença numa parte do corpo, está entrando em contato com a memória psicoafetiva contida nesta região.

A TM é uma modalidade terapêutica relativamente nova, porém a mesma apresenta normas estruturadas e amparadas pela Associação Brasileira de Terapeutas Morfoanalistas. Esta foi constituída em 2002, com Estatuto, Regimento Interno e Código de Ética próprio, administrados por Conselho Deliberativo eleito a cada dois anos. A TM pode ser realizada por fisioterapeutas e psicólogos, além de outros profissionais da área da saúde, porém, é necessário que o 
profissional realize a formação completa em TM, com quatro anos de duração.

A morfoanálise tem várias indicações, para a busca de reequilíbrio corporal, emocional e auto-conhecimento, atuando como coadjuvante no tratamento [...] O atendimento é feito através de sessões individuais de terapia morfoanalítica, em média uma vez por semana, com duração de uma hora (Associação Brasileira de Terapeutas Morfoanalistas [ABTM], 2018).

\section{Método}

\section{Caracterização do estudo e aspectos éticos}

Por se tratar de Estudo de Caso resultante de pesquisa de Pós-Doutorado do primeiro autor, necessitou da aprovação do Comitê de Ética das duas instituições (CAAE: 46822615.2.0000.5402 e CAAE: 46822615.2.3001.5415). Previamente a primeira avaliação, todos os participantes do estudo receberam esclarecimentos sobre os objetivos e procedimentos do mesmo e assinaram o Termo de Consentimento Livre e Esclarecido. Os procedimentos utilizados nesta pesquisa estão de acordo com a Resolução $\mathrm{n}^{\circ}$ 466/2012 do Conselho Nacional de Saúde.

\section{Sujeitos e critérios de seleção}

A pesquisa foi composta por dois indivíduos com transtornos de sintomas somáticos, com idade superior a 18 anos. Para inclusão no estudo, os indivíduos não podiam apresentar patologias neurológicas e/ ou ortopédicas de origem conhecida, bem como, não deviam ser tabagistas e apresentar doenças respiratórias, arritmias, marcapasso, transplante cardíaco ou fazer uso de medicamentos que influenciem diretamente a atividade autonômica do coração.

Os pacientes selecionados para este estudo foram diretamente encaminhados por fisioterapeutas pertencentes a instituição de trabalho da primeira autora, que os haviam atendido previamente e identificado que os mesmos possuíam manifestações físicas e emocionais em seus corpos, que sugeriam possível benefício da TM. Foram previamente entrevistados pela pesquisadora e então incluídos.

\section{Delineamento experimental}

Os participantes da pesquisa foram submetidos a 24 sessões de TM, realizadas uma vez por semana, com uma hora de duração. Inicialmente foi realizada uma avaliação para coleta de dados pessoais, antropométricos e da modulação autonômica. Após, foi realizada a avaliação do nível de dor e flexibilidade muscular. Além disso, responderam questionários específicos para avaliar a presença de ansiedade e depressão e investigar a presença de alexitimia. Essas avaliações foram repetidas seis dias após as sessões de número 8, 16 e 24, com exceção da modulação autonômica que foi repetida somente seis dias após a última sessão. A evolução psicoemocional do paciente foi acompanhada pelo terapeuta no decorrer de todo tratamento morfoanalítico, por meio da interpretação dos relatos.

Tanto a coleta de dados quanto as intervenções foram realizadas em sala silenciosa, com temperatura entre $21^{\circ} \mathrm{C}$ e $24^{\circ} \mathrm{C}$. Os participantes foram orientados para que antes da avaliação autonômica não fizessem uso de bebidas e/ou alimentos estimulantes como café, chá e chocolate, a fim de não influenciar o comportamento autonômico cardíaco.

\section{Dados pessoais e antropométricos}

Foram coletados nome e idade dos sujeitos e questionado a presença de comorbidades, medicamentos e substâncias em uso, como o tabaco. Para a antropometria, foram obtidos o peso e altura dos participantes por meio de balança digital com estadiômetro (Welmy ${ }^{\circledR}$, W110H, Brasil). Para o cálculo do índice de massa corporal (IMC), foi dividido o peso do indivíduo (em quilogramas) pelo quadrado da altura (em metros) (World Health Organization [WHO], 2015).

\section{Avaliação da modulação do sistema nervoso autônomo}

A modulação autonômica foi avaliada por meio da análise dos índices da VFC. Foi utilizado o Polar S810i (Polar, Finlândia), previamente validado para captação da frequência cardíaca batimento a batimento (Vanderlei, Pastre, Hoshi, Carvalho, \& Godoy, 2009; Vanderlei, Pastre, Freitas Junior, \& Godoy, 2010). Os participantes permaneceram com o Polar por 30 minutos, posicionados sobre um colchonete em decúbito dorsal, com respiração espontânea.

O software empregado para as análises dos métodos foi o Kubios HRV analysis versão 2.2 (Kubios, Bio- 
signal Analysis and Medical Image Group, Department of Physics, University of Kuopio, Finland) (Tarvainen, Niskanen, Lipponen, Ranta-aho, \& Karjalainen, 2014). A análise da VFC foi realizada por meio de métodos não lineares e lineares (analisados nos domínios do tempo e da frequência).

Métodos não lineares - Para análise da VFC por meio de métodos não lineares foi utilizada a análise quantitativa do plot de Poincaré, sendo calculados os índices SD1, SD2 e a razão SD1/SD2 (Vanderlei et al., 2009; Vanderlei et al., 2010). O SD1, representado pelo eixo transversal, corresponde ao desvio-padrão da variabilidade de curta duração, batimento-a-batimento e reflete a atividade parassimpática; o SD2, eixo longitudinal, indica o desvio padrão dos intervalos RR contínuos, representado pela variabilidade de longa duração e representa a variabilidade global (Vanderlei et al., 2010), e a razão SD1/SD2 apresenta a relação entre a variabilidade curta e longa dos intervalos RR (Vanderlei et al., 2010).

Métodos lineares - Os índices RMSSD e SDNN foram utilizados para análise da VFC no domínio do tempo. O índice de RMSSD é definido como sendo a raiz quadrada da média do quadrado das diferenças entre intervalos RR normais adjacentes, em um intervalo de tempo, expresso em milissegundos (ms) e demonstra a atividade do ramo parassimpático (Vanderlei et al., 2009). O SDNN, reflete a participação de ambos os ramos do SNA, representa o desvio-padrão da média de todos os intervalos RR normais, expresso em ms (Vanderlei et al., 2009). Para análise da VFC no domínio da frequência foram utilizados os componentes espectrais de baixa frequência ( $\mathrm{LF}-0,04$ a 0,15 $\mathrm{Hz})$, que corresponde à atuação simpática e parassimpática, porém, com predomínio simpático, e alta frequência (HF - 0,15 a 0,4 Hz) que indica atuação vagal, ambos apresentados em unidades normalizadas, que representa o valor relativo de cada componente espectral em relação à potência total menos os componentes de muito baixa frequência (Vanderlei et al., 2009). Também foi apresentada a razão entre estes componentes (LF/HF), que representa o balanço simpatovagal sobre o coração. A análise espectral foi calculada utilizando o algoritmo da transformada rápida de Fourier (Vanderlei et al., 2009).

\section{Avaliação da dor}

O nível da dor foi avaliado pela escala visual numérica $(\mathrm{EVN})$, com escala de zero a $10 \mathrm{~cm}$, sendo que o escore zero indica ausência de dor e dez denota dor insuportável (Oliveira, Batista, Pintangui, \& Araújo, 2013).

\section{Avaliação da flexibilidade}

Para avaliação da flexibilidade da cadeia muscular posterior, foi utilizado o banco de Wells, modelo instant flex (Sanny ${ }^{\circledR}$, Brasil), estabilizado contra parede, sendo os dados mensurados em centímetros. Foi realizado o teste de "Sentar e Alcançar" (Nogueira, \& Navega, 2011).

\section{Avaliação da presença de ansiedade e depressão}

A ansiedade foi avaliada pelo Inventário de Ansiedade de Beck. Este classifica a ansiedade em "grau mínimo de ansiedade", "ansiedade leve", "ansiedade moderada" ou "ansiedade severa" (Beck, Epstein, Brown, \& Steer, 1988). A depressão foi avaliada pelo Inventário de Beck para Depressão, que classifica em "ausência de depressão", "depressão leve a moderada”, "depressão moderada a grave" ou "grave depressão" (Beck, Steer, \& Garbin, 1988).

\section{Avaliação para investigar a presença de alexitimia}

Foi utilizada a versão em português da Escala de Alexitimia de Toronto (TAS), validada por Yoshida (2000), para mensurar o grau de alexitimia. Esta classifica a alexitimia como presente e ausente. Dependendo da pontuação, o resultado também pode ser inconclusivo.

\section{Intervenção com Terapia Morfoanalítica}

A sessão de TM consiste em um quadro terapêutico estruturado, porém flexível, permitindo variações de acordo com o processo terapêutico de cada um. A sessão habitual inicia em pé. Após, o paciente é convidado a se posicionar em decúbito dorsal, sendo realizada neste momento a proposta de trabalho corporal, escolhido em função da queixa física e/ou psíquica do paciente, podendo ser um trabalho postural global, que permite alongar sinergicamente as cadeias musculares de compensação, associado à respiração diafragmática, e/ou massagens superficiais e do tecido conjuntivo e vivências de consciência corporal e sensorial. Esses são realizados concomitantemente ao trabalho verbal analítico, em todas as fases da sessão. 
Ao final, em pé, o paciente é convidado a perceber as novas referências físicas e psíquicas (Pachioni et al., 2014, Pachioni et al., 2016).

\section{Avaliação analítica das sessões - Clínico-qualitativa}

Alguns relatos verbais das sessões foram transcritos, possibilitando o acompanhamento da evolução psicoemocional do sujeito durante os seis meses de intervenção. A fim de preservar o anonimato do sujeito da pesquisa, dados biográficos não relevantes foram alterados.

\section{Análise estatística}

A caracterização da população foi feita de maneira descritiva e os resultados apresentados em médias, desvios-padrão, números absolutos e percentuais. Os resultados dos índices da VFC foram apresentados por valores absolutos de cada indivíduo.

\section{Resultados qualitativos}

\section{Relato de Caso 1 - Luana}

Luana tem 35 anos, é professora, casada há oito e tem um filho de um ano de idade. Na entrevista, relata ter crises de enxaqueca e depressão. Fez terapia algumas vezes, mas não deu prosseguimento. Tomou medicamentos antidepressivos, esporadicamente. Porém, resiste ao uso dos mesmos, pois sua mãe não concorda que ela precise. Relata ter baixa autoestima e ter sofrido bullying na escola.

Apresenta estrutura física mediana, sendo alta, porém com estrutura óssea pequena nas extremidades. Apresenta quadril e seios largos, hiperextensão dos joelhos e olhar para baixo. A característica corporal de Luana lembra uma estrela, com centro compacto e extremidades frágeis e pouco sustentadoras.

Logo nas primeiras sessões, enquanto eu realizava os trabalhos corporais em Luana, observei manifestações corporais, como tensão muscular e dificuldade em soltar o peso dos segmentos de seu corpo, principalmente da cabeça. Nessas sessões, pensamentos acelerados também eram relatados por ela. Imagino que a tensão corporal de Luana, principalmente na região da musculatura cervical, dificulta a passagem de energia para a cabeça. Existe uma "tensão de passagem", somada a um medo de perder o controle.
Luana também deixa transparecer situações de conflito com os pais e irmãos e evidente simbiose com a mãe. Luana não se sente valorizada e autorizada pelos pais, mesmo em situações cotidianas. Observo sofrimento, muitas vezes profundo, como expressões de tristeza e choro durante seus relatos.

Meus pais querem controlar tudo e falam que eu não faço nada certo. Minha mãe sempre fala que eu sinto errado as coisas. Minha mãe não me deixa ter sentimentos de tristeza. Se eu não estou sorrindo e feliz todos os dias, eu estou sentindo errado. Queria que minha mãe me aceitasse como eu sou (Luana).

Mesmo, muitas vezes, complexos e ambivalentes, os sentimentos são genuínos e verdadeiros. $\mathrm{O}$ fato de sua mãe, insistentemente, não aceitar os sentimentos de Luana a deixa confusa, dificultando seu crescimento psíquico. Luana permanece num ciclo vicioso de simbiose, dependência e confusão psíquica.

Mesmo já estando na idade adulta, Luana se comporta como uma criança com medo da mãe, sendo emocionalmente dependente dela. Luana teme entrar em conflito com a mãe e perder seus cuidados. Lembro-me de seus braços finos e interpreto como medo de entrar em conflito. Medo de brigar. Voltando a imagem da estrela, penso em energia concentrada no centro, estagnada, sem canais de derivação, deixando pouca energia para as extremidades. As pernas finas podem indicar pouca troca com a terra.

No desenrolar das sessões, Luana relata situações de grandes brigas com os pais, de demissão do marido, de dificuldades dos cuidados com seu filho, tendo que driblar a intrusão dos pais e seu medo da repetição de padrões com seu filho. Luana demonstra muita angústia, porém com certo conformismo, como se já estivesse fadada a vivenciar tais situações.

Realizo diversos trabalhos corporais, dentro do quadro estruturado da TM, como, por exemplo, o lifting da cabeça, no qual posiciono minhas mãos sob sua cabeça e solicito que a paciente solte o peso desta, enquanto sustento sua cabeça fazendo pequenos movimentos da cervical, e o microestiramento cervical - trabalho de alongamento suave e progressivo da musculatura da região cervical. A princípio, Luana tem muita dificuldade em confiar e deixar soltar o peso de sua cabeça em minhas mãos. Também tem dificuldade em permitir que sua mus- 
culatura cervical relaxe para que o alongamento cervical se concretize de maneira eficaz. Com o decorrer das sessões ela vai permitindo se entregar aos meus cuidados e sua confiança, em mim e em si mesma, vai aumentando. Assim, Luana, pouco a pouco, passa a expressar melhora psicoemocional, diminuindo seus pensamentos acelerados durante a sessão e relatando frases positivas após os trabalhos corporais, como por exemplo: "Eu queria ter um tempo a mais para cuidar de mim..."; "Eu cresci"; "Estou feliz e tomando algumas decisões". Porém, mesmo com uma estrutura psicocorporal mais fortalecida, suportando melhor as adversidades que vão chegando, Luana ainda se boicota para manter a composição neurótica.

Acredito que o fortalecimento emocional de Luana resulte em maior posicionamento frente aos seus pais. Após "explosões" com seus pais, Luana decide procurar um médico psiquiatra a fim de buscar auxílio medicamentoso. Luana relata: "Eu não gosto de tomar esses remédios, mas eu não quero mais ter as crises que eu tive. Fiquei assustada com minha última crise e não quero que meu filho me veja assim".

Sinto que o trabalho psicocorporal deu suporte para ela procurar e aceitar auxílio medicamentoso para, assim, com associação de métodos terapêuticos, se fortalecer mais rapidamente e conseguir caminhar rumo a sua individuação. Luana decide mudar e começa com medicamento antidepressivo após a $18^{\mathrm{a}}$ sessão de terapia morfoanalítica.

Com o ritmo semanal dos trabalhos psicocorporais, Luana foi encontrando um tempo e lugar para se sentir plenamente, experimentando manifestações físico-emocionais positivas, como melhora da postura corporal, relaxamento e expressões de alegria e entusiasmo. Ao final das 24 sessões, Luana estava mais "inteira", mais plena e independente. Não tem mais os pensamentos acelerados durante a sessão. É visível a melhora de Luana. Ela está feliz e animada. Está se permitindo "perder tempo" assistindo desenhos com o filho. E quase não fala mais da mãe nas últimas sessões.

\section{Relato de Caso 2 - Solange}

Solange tem 47 anos e trabalha na rede pública. Foi casada por 10 anos e está separada há 10 anos. Tem dois filhos, de 14 anos e 12 anos. O filho de 14 anos apresenta síndrome de pânico, teve a primeira crise logo após a separação dos pais e aos 10 anos foi diagnosticado com síndrome de pânico. Solange teve uma infância difícil, com dificuldades financeiras. Seu pai era ausente e tinha várias amantes. Perdeu a mãe aos 18 anos. Casou-se cedo, com um homem de comportamento agressivo. Atualmente, seu pai apresenta depressão. Por ser a única mulher entre vários irmãos, desde a morte de sua mãe ela se sente responsável pela família inteira. Assumiu uma carga maior do que seu ego era capaz de suportar. Paradoxalmente, mesmo com várias questões pessoais difíceis e profundas, Solange está sempre "de bem com a vida" otimista e sorridente.

Apresenta estrutura física mediana e me dá a impressão de uma pessoa compacta (musculatura firme). Tem ombros e cintura estreitos, porém suas pernas são grossas. Chama a atenção o volume de suas panturrilhas, sendo até desproporcional em relação a sua estrutura corporal. Acredito que o sobrepeso observado em sua avaliação física seja decorrente do volume de suas pernas.

Quando se posiciona no setting, apresenta base de sustentação alargada, pernas rodadas externamente e joelhos em valgo (próximos um ao outro). Normalmente cruza as mãos em frente ao corpo, me remetendo a imagem de uma menina envergonhada e solitária.

Logo na primeira sessão, durante os toques clássicos de uma sessão de terapia morfoanalítica, sinto a musculatura do trapézio muito tensa e dura. Me remete a um tijolo. Acho que é um dos corpos mais duros que já toquei. Realizo um trabalho respiratório, chamando a atenção para os toques corporais, em seguida, realizo o microestiramento cervical. No espaço verbal, Solange relata:

É difícil sentir meu corpo. Minha respiração é muito presa. É difícil respirar. Normalmente eu paro de respirar e fico no piloto automático. E eu não paro para pensar no meu corpo. Eu acordo, coloco a roupa e saio. Não tenho tempo de pensar. Mas, gostei deste trabalho. Tive um tempo para sentir e pensar no meu corpo (Solange).

Finalizo a sessão com uma massagem suave em seus braços e pernas, com o intuito de oferecer continência ao seu vazio interior. Após a massagem, ela relata: "Nossa! Eu estou leve! Na hora que você ia passando a mão ia tirando todo o peso. Quando chegou até o meu pé parece que o peso foi saindo". 
Durante as sessões, observando a fisionomia de Solange, percebo que ela sente muita dor, principalmente em sua coluna vertebral e membros inferiores, porém reclama muito pouco. Já está acostumada! Ademais, a mesma relatou valores elevados para a dor na avaliação inicial. A expressão "piloto automático" me remete a uma pessoa que faz, faz e faz, sem pensar, sem presença. Parece demonstrar pouca consciência do seu sentir, do seu perceber. Os sentimentos vividos não são pensados e simbolizados, mas sim descarregados em ato motor. Isto vai ao encontro da presença de alexitimia detectada em sua avaliação inicial.

Nas sessões subsequentes, percebo que, em momentos de visível tensão corporal, nos seus relatos, Solange costuma rir em vez de chorar. A expressão "piloto automático" continua bem presente nos momentos iniciais das sessões. Este estado de piloto automático demonstra desconexão de seu corpo e de seus sentimentos, não conseguindo sentir a sua dor. $\mathrm{O}$ caminho que seu corpo encontra como saída é somatizar a dor que não pode ser vivida. No decorrer das sessões, através dos toques corporais é possível acessar sensações e sentimentos, até então desconhecidos para ela, como por exemplo, uma raiva velada.

Em várias sessões, quando questionada sobre suas sensações após os toques corporais, é comum Solange responder: "Não senti nada de ruim"; "Sua mão não é pesada”; "Sua mão não aperta”. Isso sempre me intrigou. Lembro que, desde as sessões iniciais, no momento que eu toco seu pescoço para realizar alongamento cervical, percebia espasmos na musculatura do ombro e pescoço. Isso sugere um corpo em sobressalto, um corpo preocupado em receber agressão. Na $13^{a}$ sessão, eu já sentia uma relação transferencial suficiente para aprofundar um pouco mais e insisti para que ela falasse um pouco mais. Solange responde: "É uma mão que não me agride". Sinto que seu corpo duro e compacto esconde situações agressivas vividas por ela, podendo justificar os sobressaltos observados em seu corpo. Nesta sessão ela revive uma situação de abuso de muitos anos atrás, expressando muito sofrimento e raiva, guardados "a sete chaves" em seu corpo. Esta experiência vivida estava apagada de sua memória atual. A partir da interiorização do que é bom, Solange se fortalece também para sentir o ruim. Seu corpo, até então adormecido, é acordado para experienciar as vicissitudes da vida - é acordado para sentir "a dor e a delícia de ser o que é”, parafraseando Caetano Veloso em uma de suas canções.
Nas sessões subsequentes ela modifica a resposta ao meu toque, respondendo: "É bom"; "Sua mão é agradável e leve”. Em associação, manifestações positivas de relaxamento, alívio e alegria passaram a ser mais frequentes. Também percebo melhora em seu padrão postural com diminuição da base de sustentação e melhora nas dores corporais. Pouco a pouco, Solange vai se nutrindo com os trabalhos psicocorporais, vai se permitindo ter um tempo maior para ela e conseguindo soltar seus pesos emocionais.

A expressão "piloto automático", muitas vezes, utilizada por Solange desde a primeira sessão, aparece agora com menor frequência. Essa expressão denota a ideia de um corpo, muitas vezes, automatizado e sem mente sem piloto. Com o decorrer do trabalho morfoanalítico, o termo "piloto automático" vai se ressignificando. Solange apresenta, agora, maior integração mente-corpo.

No início, apesar de sua vida sofrida, ela relata os fatos sorrindo e, às vezes, brincando com a situação. Um riso nervoso. De início, Solange tem desconexão entre expressão emocional e experiência. A evolução do trabalho permite que Solange possa se ressignificar, mudando a visão de si mesma, podendo relacionar sorriso com alegria. Isso pode ser claramente observado em sua resposta em relação a alexitimia, observada em sua avaliação inicial e ausente na final.

\section{Resultados quantitativos}

Foram avaliadas duas pacientes Luana e Solange, com idade 35 e 47 anos, respectivamente. Ambas apresentam sobrepeso, Luana com IMC de $28 \mathrm{~kg} / \mathrm{m}^{2}$ e Solange com IMC de $26 \mathrm{~kg} / \mathrm{m}^{2}$.

Os valores referentes ao nível de dor, flexibilidade e sintomas de alexitimia, ansiedade e depressão são apresentados nas Tabelas 1 e 2 .

A Tabela 3 apresenta os valores dos índices da VFC nos domínios do tempo, da frequência e plot de Poincaré de ambas as pacientes nos momentos inicial e final ao tratamento.

\section{Discussão}

A presente pesquisa foi realizada com duas pacientes submetidas a 24 sessões de TM, com inventários realizados antes, durante e após a finalização das sessões, cujos dados estão discutidos a seguir.

A fim de facilitar ao leitor a visualização, em suas grandes linhas, das características de personalidade mais marcantes de cada paciente, foram escolhidos 


\section{Tabela 1}

Nível de dor, valores da flexibilidade e sintomas de alexitimia, ansiedade e depressão da paciente Luana. Índices da avaliação inicial, duas intermediárias e uma final.

\begin{tabular}{|c|c|c|c|c|}
\hline & 1a Avaliação & 2a Avaliação & 3a Avaliação & 4a Avaliação \\
\hline \multicolumn{5}{|l|}{ Dor } \\
\hline Cabeça & 10,0 & 9,0 & 8,0 & 3,0 \\
\hline Ombro Direito & 4,0 & 0,0 & 0,0 & 0,0 \\
\hline Pé Esquerdo & 5,0 & 5,0 & 3,0 & 7,0 \\
\hline \multicolumn{5}{|l|}{ Flexibilidade } \\
\hline Banco de Wells & 9,5 & 7,5 & 7,8 & 10,0 \\
\hline \multicolumn{5}{|c|}{ Sintomas Emocionais } \\
\hline Alexitimia - TAS & $\begin{array}{c}80,0 \\
\text { (presença de } \\
\text { alexitimia) }\end{array}$ & $\begin{array}{c}87,0 \\
\text { (presença de } \\
\text { alexitimia) }\end{array}$ & $\begin{array}{c}82,0 \\
\text { (presença de } \\
\text { alexitimia) }\end{array}$ & $\begin{array}{c}\quad 64,0 \\
\text { (ausência de } \\
\text { alexitimia) }\end{array}$ \\
\hline Ansiedade - Beck & $\begin{array}{c}26,0 \\
\text { (ansiedade severa) }\end{array}$ & $\begin{array}{c}33,0 \\
\text { (ansiedade severa) }\end{array}$ & $\begin{array}{c}19,0 \\
\text { (ansiedade } \\
\text { moderada) }\end{array}$ & $\begin{array}{c}\text { 3,0 } \\
\begin{array}{c}\text { (grau mínimo de } \\
\text { ansiedade) }\end{array}\end{array}$ \\
\hline Depressão - Beck & $\begin{array}{c}27,0 \\
\text { (depressão de } \\
\text { moderada a grave) }\end{array}$ & $\begin{array}{c}27 \\
\text { (depressão grave) }\end{array}$ & $\begin{array}{c}19,0 \\
\text { (depressão de } \\
\text { moderada a grave) }\end{array}$ & $\begin{array}{c}4,0 \\
\text { (ausência de } \\
\text { depressão ou } \\
\text { depressão mínima) }\end{array}$ \\
\hline
\end{tabular}

Tabela 2

Nível de dor, valores da flexibilidade e sintomas de alexitimia, ansiedade e depressão da paciente Solange. Índices da avaliação inicial, duas intermediárias e uma final.

\begin{tabular}{|c|c|c|c|c|}
\hline & 1a Avaliação & 2a Avaliação & $3^{a}$ Avaliação & $4^{\text {a Avaliação }}$ \\
\hline \multicolumn{5}{|l|}{ Dor } \\
\hline Coluna & 8,0 & 5,0 & 4,0 & 4,0 \\
\hline Pernas & 7,0 & 4,0 & 3,0 & 3,0 \\
\hline \multicolumn{5}{|l|}{ Flexibilidade } \\
\hline Banco de Wells & 4,0 & 5,7 & 19,0 & 19,5 \\
\hline \multicolumn{5}{|c|}{ Sintomas Emocionais } \\
\hline Alexitimia-TAS & $\begin{array}{c}75,0 \\
\text { (presença de } \\
\text { alexitimia) }\end{array}$ & $\begin{array}{l}66,0 \\
(?)\end{array}$ & $\begin{array}{l}68,0 \\
(?)\end{array}$ & $\begin{array}{c}56,0 \\
\text { (ausência de } \\
\text { alexitimia) }\end{array}$ \\
\hline Ansiedade - Beck & $\begin{array}{c}14,0 \\
\text { (ansiedade leve) }\end{array}$ & $\begin{array}{l}\text { 7,0 } \\
\text { (grau mínimo de } \\
\text { ansiedade) }\end{array}$ & $\begin{array}{c}5,0 \\
\text { (grau mínimo de } \\
\text { ansiedade) }\end{array}$ & $\begin{array}{c}6,0 \\
\text { (grau mínimo de } \\
\text { ansiedade) }\end{array}$ \\
\hline Deprssão-Beck & $\begin{array}{c}8,0 \\
\text { (ausência de } \\
\text { depressão ou } \\
\text { depressão mínima) }\end{array}$ & $\begin{array}{c}3,0 \\
\text { (ausência de } \\
\text { depressão ou } \\
\text { depressão mínima) }\end{array}$ & $\begin{array}{c}3,0 \\
\text { (ausência de } \\
\text { depressão ou } \\
\text { depressão mínima) }\end{array}$ & $\begin{array}{c}3,0 \\
\text { (ausência de } \\
\text { depressão ou } \\
\text { depressão mínima) }\end{array}$ \\
\hline
\end{tabular}

pseudônimos que reflitam boa representação simbólica das características de ambas.

O nome Luana, com o prefixo Lua, remete a uma pessoa introspectiva, com diferentes "fases da Lua".
Iniciou suas sessões na fase Cheia, quase explodindo, no decorrer destas, deixou transparecer características Minguantes, com risco de implosão, a seguir foi-se renovando, finalizando suas sessões em estado Cres- 
Tabela 3

Comparação dos índices da Variabilidade da Frequência Cardíaca (VFC) no domínio do tempo, da frequência e índice geométrico (plot de Poincaré), nos momentos antes e depois de 6 meses de tratamento. Dados apresentados em valores absolutos.

\begin{tabular}{lcccc}
\hline & $\begin{array}{c}\text { Luana } \\
\text { Antes }\end{array}$ & $\begin{array}{c}\text { Luana } \\
\text { Depois }\end{array}$ & $\begin{array}{c}\text { Solange } \\
\text { Antes }\end{array}$ & $\begin{array}{c}\text { Solange } \\
\text { Depois }\end{array}$ \\
\hline Mean RR(ms) & 824,000 & 938,200 & 811,000 & 952,900 \\
Domínio do tempo & & & & \\
RMSSD (ms) & 18,800 & 41,400 & 11,200 & 14,800 \\
SDNN (ms) & 42,200 & 47,600 & 31,500 & 24,100 \\
Domínio da frequência & & & & \\
LF (un) & 78,000 & 61,200 & 67,700 & 49,300 \\
HF (un) & 21,600 & 38,500 & 32,300 & 50,600 \\
LF/HF (ms) & 3,607 & 1,590 & 2,099 & 0,974 \\
Plot de Poincaré & & & & \\
SD1 (ms) & 13,300 & 29,300 & 7,900 & 10,500 \\
SD2 (ms) & 58,200 & 60,500 & 43,900 & 32,400 \\
SD1/SD2 (ms) & 0,228 & 0,484 & 0,180 & 0,324 \\
\hline
\end{tabular}

Mean RR: média dos intervalos RR; ms: milissegundos; $\mathrm{ms}^{2}$ : milissegundos ao quadrado; un: unidades normalizadas; RMSSD: raiz quadrada da média do quadrado das diferenças entre intervalos RR normais adjacentes, em um intervalo de tempo, expresso em milissegundos SDNN: desvio-padrão da média de todos os intervalos RR normais, expresso em milissegundos; LF: Componente de baixa frequência; HF: Componente de alta frequência; LF/HF: relação Componente de baixa frequência/ Componente de alta frequência. SD1: desvio-padrão da variabilidade instantânea batimento-a-batimento; SD2: desvio-padrão a longo prazo dos intervalos RR contínuos; SD1/SD2: razão entre as variações curta e longa dos intervalos RR.

cente. O nome Solange, com o prefixo Sol, remete a uma pessoa iluminada, quente e alegre, "faça sol ou faça chuva"! Mesmo com a vida marcada por intempéries, ela se mantém firme, por vezes com mais ou com menos brilho.

As queixas principais apresentadas na avaliação inicial diferem substancialmente. Luana relatava principalmente vivências emocionais ("Não consigo relaxar"), enquanto Solange predominantemente sensações físicas ("Tenho muita dor no osso da perna e na coluna"). Ambas as pacientes apresentam sobrepeso.

Os resultados obtidos nas características físicas avaliadas da paciente Luana evidenciaram melhora da dor, sendo que as dores de cabeça melhoraram em $70 \%$. Quanto a flexibilidade corporal, foi observada apenas uma discreta melhora, embora ainda permaneça pouco flexível. Na avaliação pelos questionários foi evidenciada melhora tanto na alexitimia, quanto na ansiedade e depressão. A alexitimia, presente na avaliação inicial, estava ausente ao final das 24 sessões de atendimento. A ansiedade, avaliada inicialmente como severa, apresentou-se com grau mínimo. A depressão passou de moderada/grave para depres- são mínima ou ausente. A melhora nos quesitos dos três questionários após as sessões pode ser resultante do trabalho psicocorporal, porém o fato da alexitimia ter se tornado ausente pode influenciar na melhora do quadro emocional, visto que, ao reconhecer suas emoções de maneira adequada, o indivíduo reduz a somatização e consequentemente a exposição aos sintomas de ansiedade e depressão.

É importante salientar que, embora Luana tenha iniciado tratamento medicamentoso na $19^{\mathrm{a}}$ sessão, a $3^{a}$ avaliação foi realizada antes disso. Nesta avaliação já foi evidenciada melhora substancial da ansiedade e depressão, passando, respectivamente, de severa e grave para moderada.

Os resultados obtidos nas características físicas avaliadas da paciente Solange evidenciaram melhora aproximada de $50 \%$ da dor nas regiões da coluna e pernas. Quanto a flexibilidade corporal, foi observado um grande aumento na flexibilidade da cadeia muscular posterior, sendo o índice final aproximadamente cinco vezes maior que o inicial. Na avaliação pelos questionários foi evidenciada melhora mais acentuada na alexitimia, passando de presente para 
ausente. A ansiedade, inicialmente leve, passou para mínima e a depressão continuou ausente ou mínima, após 24 sessões de atendimento.

A TM, assim como no presente estudo, tem mostrado melhora para pacientes em outras condições clínicas. Em uma intervenção com 16 sessões de TM em pacientes pós cirurgia de câncer de mama foi observada melhora estatisticamente significativa na flexibilidade (Pachioni et al., 2014), na depressão e autoestima (Pachioni et al., 2016).

Foi observada evidente melhora nas características posturais das pacientes, após as sessões de TM, como maior alinhamento corporal, horizontalização do olhar e diminuição na hiperextensão de joelhos e da base de sustentação. Esta melhora pode ser compreendida pela sincronia do trabalho mente-corpo com a relação entre personalidade e postura, pois o estado emocional pode ser causa ou consequência de alterações posturais (Canales, Cordás, Fiquer, Cavalcante \& Moreno, 2010).

Tanto Luana, quanto Solange apresentaram níveis elevados de dor em sua avaliação inicial, podendo esta ser intensificada pela presença de alexitimia. As técnicas utilizadas na TM são propícias para o despertar da consciência corporal e a experienciação de sentimentos (Pachioni et al., 2016). A prestação de cuidados alternativos, como a autoconsciência emocional e o estímulo ao indivíduo na identificação de seus sentimentos, pode auxiliar no processamento da dor (Margalit et al., 2014).

A presença de Alexitimia em ambas as pacientes identifica um traço da personalidade dessas, caracterizado pela dificuldade em identificar e reconhecer suas próprias emoções (Yoshida, 2000). Com o decorrer do trabalho morfoanalítico observa-se, nas pacientes, maior capacidade para a identificação das suas emoções. O indivíduo que sabe compreender e expressar seus estados emocionais lida melhor com experiências negativas e apresenta melhores respostas de adaptação (Hozoori, \& Barahmand, 2013).
Ao analisar as modulações autonômicas da paciente Luana, verificou-se acentuado aumento nos índices RMSSD, HF e SD1, que representam a atividade parassimpática. No índice LF, que representa atividade simpática, foi evidenciada uma diminuição. Os índices SDNN e SD2, que representam a variabilidade global, se apresentaram com um discreto aumento. A razão LF/HF, que representa a relação simpático/parassimpático, mostrou-se mais próxima de um.

Ao analisar as modulações autonômicas da paciente Solange, verificou-se aumento nos índices RMSSD, HF e SD1 (índices parassimpáticos), embora bem menos acentuado, quando comparado a paciente Luana. No índice LF (simpático), foi evidenciada uma diminuição. Os índices SDNN e SD2 (variabilidade global), se apresentaram discretamente diminuídos. A razão LF/HF, que representa a relação simpático/ parassimpático, mostrou-se mais próxima de um.

Luana, com mais comprometimento autonômico, obteve melhora mais acentuada. Solange, com menos comprometimento, obteve melhora menos expressiva.

O sistema nervoso autônomo, simpático e parassimpático, avaliado pelos índices da VFC, fornece um indicador sensível e antecipado da saúde humana. Assim, permite interpretações interessantes das funções internas do organismo, tanto em condições normais quanto patológicas (Vanderlei et al., 2009). Maiores valores do tônus parassimpático podem estar relacionados ao bem-estar psicológico, como sentimentos de alegria e serenidade, emotividade positiva e engajamento social (Kemp, \& Quintana, 2013).

Com base nos resultados, podemos verificar evidente melhora em ambas as pacientes, após 24 sessões de TM. Essa eficácia foi observada tanto nos índices emocionais, quanto nos físicos. Em termos de integração psicossomática, observam-se como as melhoras no campo mental e físico se entrelaçam. Isso demonstra a efetividade desta modalidade terapêutica, que associa os trabalhos corporais com o trabalho verbal analítico, na melhor integração psicossomática.

\section{Referências}

American Psychiatric Association - APA. (2014). DSM-5: Manual diagnóstico e estatístico de transtornos mentais. Porto Alegre, RS: Artmed. Recuperado de https://aempreendedora.com.br/wp-content/uploads/2017/04/ Manual-Diagn\%C3\%B3stico-e-Estat\%C3\%ADstico-de-Transtornos-Mentais-DSM-5.pdf

Angenendt, J., \& Härter, M. (2015). Somatoform disorders. In J. Wright (Ed.), International encyclopedia of the social \& behavioral sciences (IESBS) (2a ed., pp. 10-15). London: Elsevier. 
Associação Brasileira de Terapeutas Morfoanalistas - ABTM. (2018). Home. Mogi Mirim, SP: o autor. Recuperado de: http://www.abtm.com.br/

Ávila, L. A. (2002). Doenças do corpo e doenças da alma. São Paulo, SP: Escuta.

Ávila, L. A. (2012). O corpo, a subjetividade e a psicossomática. Tempo Psicanalítico, 44(1), 51-69.

Beck, A. T., Epstein, N., Brown, G., \& Steer, R. A. (1988). An inventory for measuring clinical anxiety: The beck anxiety inventory. Journal of Consulting and Clinical Psychology, 56(6), 893-897. http://doi.org/10.1037//0022006x.56.6.893

Beck, A. T., Steer, R. A., \& Garbin, M. G. (1988). Psychometric properties of the beck depression inventory: Twenty-five years of evaluation. Clinical Psychology Review, 8(1), 77-100. https://doi.org/10.1016/0272-7358(88)90050-5

Canales, J. Z., Cordás, T. A., Fiquer, J. T., Cavalcante, A. F., \& Moreno, R. A. (2010). Posture and body image in individuals with major depressive disorder: A controlled study. Revista Brasileira de Psiquiatria, 32(4), 375-380. https://doi.org/10.1590/S1516-44462010000400010

Castro, M. G., Andrade, T. M. R., \& Muller, M. C. (2006). Conceito mente e corpo através da história. Psicologia em Estudo, 11(1), 39-43. https://doi.org/10.1590/S1413-73722006000100005

Chalah, M. A., \& Ayache, S. S. (2017). Alexithymia in multiple sclerosis: A systematic review of literature. Neuropsychologia, (104), 31-47. https://doi.org/10.1016/j.neuropsychologia.2017.07.034

Diefenbach, N. (2003). O “Eu corporal” em terapia morfoanalítica. Fisioterapia em Movimento, 16(2),73-82.

Fernandes, M. H. (2011). Corpo. São Paulo, SP: Casa do Psicólogo.

Fontes, I. (2006). A ternura tátil: O corpo na origem do psiquismo. Psiquê, 10(17), 109-120.

Heidari, Z., Feizi, A., Roohafza, H., Hassanzadeh, K. A., \& Adibi, P. (2017). Somatoform symptoms profiles in relation to psychological disorders: A population classification analysis in a large sample of general adults. Psychiatry Research, 254, 173-178. https:// doi.org/10.1016/j.psychres.2017.04.064

Hozoori, R., \& Barahmand, U. (2013). A study of the relationship of alexithymia and dissociative experiences with anxiety and depression in students. Procedia: Social and Behavioral Sciences, 84, 128-133. https://doi.org/10.1016/j.sbspro.2013.06.522

Kemp, A. H., \& Quintana, D. S. (2013). The relationship between mental and physical health: Insights from the study of heart rate variability. International Journal of Psychophysiology, 89(3), 288-296. https://doi.org/10.1016/j.ijpsycho.2013.06.018

Krygier, J. R., Heathers, J. A. J., Shahrestani, S., Abbot, M., Gross, J. J., \& Kemp, A. H. (2013). Mindfulness meditation, well-being, and heart rate variability: A preliminary investigation into the impact of intensive vipassana meditation. International Journal of Psychophysiology, 89(3), 305-313. https://doi.org/10.1016/j.ijpsycho.2013.06.017

Kuehl, L. K., Deuter, C. E., Richter, S., Schultz, A., Rüddel, H., \& Schächinger, H. (2015). Two separable mechanisms are responsible for mental stress effects on high frequency heart rate variability: An intra-individual approach in a healthy and a diabetic sample. International Journal of Psychophysiology, 95(3), 299-303. https://doi.org/10.1016/j.ijpsycho.2014.12.003

Livorato, A. (2004). Corpo, coração e consciência: Terapia morfoanalítica. In Convenção Brasil Latino Américano, Congresso Brasileiro, Encontro Paranaense de Psicoterapias Corporais, Anais do 1 Convenção Brasil e Latino Américano, 4 Congresso Brasileiro e 9 Encontro Paranaense de Psicoterapias Corporais. Curitiba, PR: Centro Reichiano.

Margalit, D., Ben Har, L., Brill, S., \& Vatine, J. J. (2014). Complex regional pain syndrome, alexithymia, and psychological distress. Journal of Psychosomatic Research, 77(4), 273-277.https://doi.org/10.1016/j.jpsychores.2014.07.005

Mignard, P. (2010). Da sensação de verticalidade ao sentimento de prumo na vida. Revista Brasileira de Terapia Morfoanalítica, (1), 9-15.

Nogueira, H. C., \& Navega, M. T. (2011). Influência da escola de postura na qualidade de vida, capacidade funcional, intensidade de dor e flexibilidade de trabalhadores administrativos. Fisioterapia \& Pesquisa, 18(4), 353-358. https:// doi.org/10.1590/S1809-29502011000400010

Oliveira, V. M. A., Batista, L. S. P., Pintangui, A. C. R., \& Araújo, R. C. (2013). Efeito do kinesio taping na dor e discinesia escapular em atletas com síndrome do impacto do ombro. Revista Dor, 14(1),27-30. https:// doi.org/10.1590/S1806-00132013000100007 
Pachioni, F. S. M., Fregonesi, C. E. P. T., \& Mantovani, A. M. (2014). O efeito da terapia morfoanalítica na qualidade de vida, estresse e flexibilidade de mulheres pós-cirurgia de câncer de mama. Manual Therapy Posturology \& Rehabilitation Journal, (12), 95-114. https://doi.org/10.17784/mtprehabjournal.2014.12.167

Pachioni, F. S. M., Palma, M. R., Mantovani, A. M., \& Fregonesi, C. E. P. T. (2016). Morfoanalitica therapy as a therapeutic resource post breast cancer surgery for women. Manual Therapy Posturology \& Rehabilitation Journal, (14), 1-5. https://doi.org/10.17784/mtprehabJournal.2016.14.293

Peyrot, S., Mignard, P., \& Albuquerque, L. C. (2005). Terapia morfoanalítica. In: A. R. Ribeiro, F. A. Souza, \& R. Magalhães. Catálogo de abordagens terapêuticas (pp. 255-258). São Paulo, SP: Casa do Psicólogo.

Souza, M. Z. (2016). Entre parâmetros e certezas da avaliação osteopática. São Paulo, SP: Idot.

Tang, N. K. (2017). Cognitive behavioural therapy in pain and psychological disorders: Towards a hybrid future. Progress in Neuro-psychopharmacology \& Biological Psychiatry, 87(part B), $281-289$. https://doi.org/ 10.1016/j.pnpbp.2017.02.023

Tarvainen, M. P., Niskanen, J. P., Lipponen, J. A., Ranta-aho, P. O., \& Karjalainen, P. A. (2014). Kubios HRV: Heart rate variability analysis software. Computer Methods and Programs Biomedicine, 113(1), $210-220$. https://doi.org/10.1016/j.cmpb.2013.07.024

Tófoli, L. F., Andrade, L. H., \& Fontes, S. (2011). Somatização na América Latina: Uma revisão sobre a classificação de transtornos somatoformes, síndromes funcionais e sintomas sem explicação médica. Revista Brasileira de Psiquiatria, 33(Sup.1), S59-S69. https://doi.org/10.1590/S1516-44462011000500006

Vanderlei, L. C. M., Pastre, C. M., Freitas, I. F., Jr, \& Godoy, M. F. (2010). Geometric indexes of heart rate variability in obese and eutrophic children. Arquivos Brasileiros Cardiologia, 95(1), 35-40. https://doi.org/10.1590/S0066-782X2010005000082

Vanderlei, L. C. M., Pastre, C. M., Hoshi, R. A., Carvalho, T. D., \& Godoy, M. F. (2009) Noções básicas de variabilidade da freqüência cardíaca e sua aplicabilidade clínica. Revista Brasileira de Cirurgia Cardivascular, 24(2), $205-217$. https://doi.org/10.1590/S0102-76382009000200018

World Health Organization - WHO. (2015). Health topics: Obesity. Geneva: o autor. Recuperado de: http://www. who.int/topics/obesity/en/

World Health Organization - WHO. (2016). Centro colaborador da OMS para a classificação de doenças em português: Classificação estatística internacional de doenças e problemas relacionados à saúde - CID-10. Geneva: o autor. Recuperado de: http://www.datasus.gov.br/cid10/V2008/cid10.htm

Yoshida, E. M. P. (2000). Toronto Alexithymia Scale-TAS: Precisão e validade da versão em português. Psicologia: Teoria e Prática, 2(1), 59-74.

\section{Cristina Elena Prado Teles Fregonesi}

Professora Doutora do Departamento de Fisioterapia, Faculdade de Ciências e Tecnologia, Universidade Estadual Paulista Júlio de Mesquita Filho (Unesp), Presidente Prudente - SP. Brasil.

E-mail: cristina@fct.unesp.br http://orcid.org/0000-0003-0395-0899

\section{Lazslo Antonio Ávila}

Livre docente do Departamento de Psiquiatria e Psicologia Médica, Faculdade de Medicina de São José do Rio Preto (Famerp), São José do Rio Preto - SP. Brasil.

E-mail: lazslo@famerp.br http://orcid.org/0000-0001-6392-1016

Endereço de para envio de correspondência:

Rua Roberto Simonsen, 305.

Bairro: Centro Educacional. CEP: 19060-900. Presidente Prudente - SP. Brasil. 
Recebido 26/10/2017

Reformulado 06/06/2018 - 09/07/2018

Aceito 25/07/2018

Received $10 / 26 / 2017$

Reformulated 06/06/2018 - 07/09/2018

Approved 07/25/2018

Recibido $26 / 10 / 2017$

Reformulado 06/06/2018 - 09/07/2018

Aceptado 25/07/2018

Como citar: Fregonesi, C. E. P. T., \& Ávila, L. A. (2019). Terapia Morfoanalítica nos transtornos de sintomas somáticos. Psicologia: Ciência e Profissão, 39, 1-15. https://doi.org/10.1590/1982-3703003187122

How to cite: Fregonesi, C. E. P. T., \& Ávila, L. A. (2019). Morphoanalytic Therapy in somatic symptom disorders. Psicologia: Ciência e Profissão, 39, 1-15. https://doi.org/10.1590/1982-3703003187122

Cómo citar: Fregonesi, C. E. P. T., \& Ávila, L. A. (2019). Terapia Morfoanalítica en los trastornos de síntomas somáticos. Psicologia: Ciência e Profissão, 39, 1-15. https://doi.org/10.1590/1982-3703003187122 